\title{
The physics potential of the HERA-B RICH
}

P. Križan ${ }^{a} *$, I. Arinyo ${ }^{b}$, M. Atiya ${ }^{c}$, D. Broemmelsiek ${ }^{d}$, J. Carvalho $^{e}$, D. Dujmićf R. Eckmann ${ }^{\mathrm{f}}$, L. Garrido ${ }^{\mathrm{b}}$, A. Gorišek ${ }^{\mathrm{a}}$, M. Ispirian ${ }^{\mathrm{g}}$, I. Ivaniouchenkov ${ }^{\mathrm{e}}$,

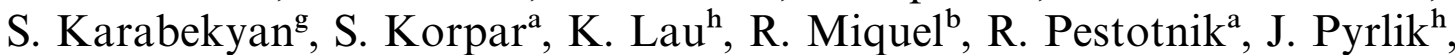

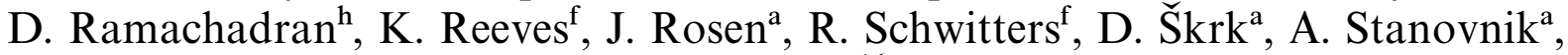
M. Starič́a, T. Živko ${ }^{a}$

${ }^{a} J$. Stefan Institute, University of Ljubljana, P.O.Box 100, Jadranska 19, 61000 Ljubljana, Slovenia

${ }^{\mathrm{b}}$ University of Barcelona, Barcelona, Spain, ${ }^{\mathrm{c}} B N L$, Upton, USA,

${ }^{\mathrm{d}}$ Northwestern University, Evanston, USA,

${ }^{\mathrm{e}}$ LIP, University of Coimbra, Coimbra, Portugal,

${ }^{\mathrm{f}}$ University of Texas, Austin, USA,

${ }^{\mathrm{g}} \mathrm{DESY}$, Hamburg, Germany,

${ }^{\mathrm{h}}$ University of Houston, Houston, USA

\begin{abstract}
The physics potential of the RICH counter of the HERA-B experiment is illustrated in several MC simulated examples. In particular, we discuss its performance as an auxiliary tracking device. (C) 1999 Elsevier Science B.V. All rights reserved.
\end{abstract}

PACS: $11.30 .-\mathrm{j} ; 29.40 .-\mathrm{n} ; 29.40 . \mathrm{ka}$

Keywords: RICH; HERA-B experiment; High-energy physics

\section{Introduction}

In the $\mathrm{CP}$ violation measurement with the HERA-B detector at the HERA $\mathrm{e}-\mathrm{p}$ collider at DESY in Hamburg [1], tagging of the B meson flavour will be performed by identification of the charged kaon with a RICH counter [2-4]. In order to efficiently separate kaons from pions up to about $50 \mathrm{GeV} / c$, the RICH has to detect about

* Corresponding author.

E-mail address: peter.krizan@ijs.si (P. Križan)
30 photons per Cherenkov ring for $\beta=1$ charged particles.

As discussed in more detail in Ref. [5], from the data accumulated in the period from August to October 1998, we could conclude that the counter behaves according to expectations. In particular, about 30 photons are expected when the radiator vessel is filled with the $\mathrm{C}_{4} \mathrm{~F}_{10}$ radiator gas, with a single photon resolution of 0.7 mrad. As a consequence, the predictions of counter performance as an identification and tracking device, discussed in what follows, become more reliable. 


\section{RICH as a particle identification device}

\subsection{Tagging with kaons}

In the $\mathrm{CP}$ violation measurement, e.g., in channels $B^{0} \rightarrow J / \psi K_{\mathrm{S}}^{0}$ and $B^{0} \rightarrow \pi^{+} \pi^{-}$, it is essential to determine the flavour of associately produced B meson. As can be seen from Figs. 1 and 2, the expected performance matches well with the momentum distribution of the tagging kaons.

\section{2. $B^{0} \rightarrow \pi^{+} \pi^{-}, \mathrm{K}^{+} \pi^{-}$}

The momentum spectrum of the pions and kaons in the two-body final states is much harder, as can be seen from Fig. 3. In these exclusive channels one

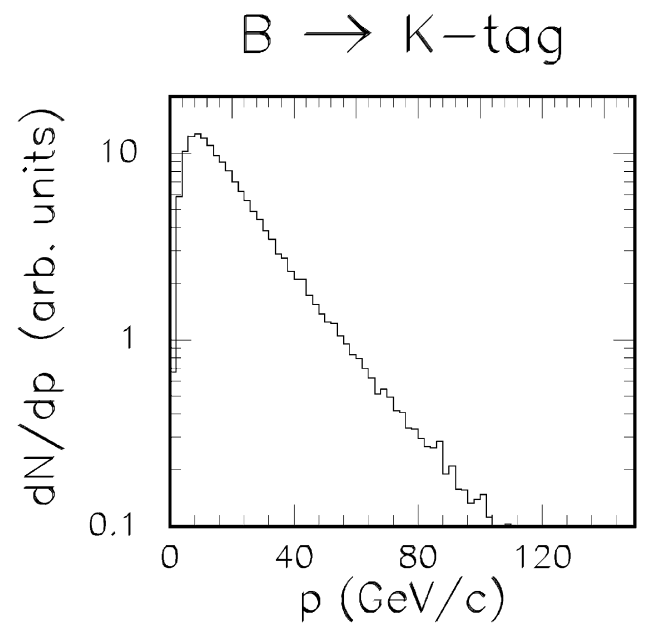

Fig. 1. Tagging kaons: momentum spectrum at HERA-B. could, however, relax the purity requirements in order to increase the efficiency (see Fig. 4).

\section{RICH as an auxiliary tracking device}

In the winter 1998 and spring 1999 run periods, only a very limited part of the tracking system will be available, so that the physics programme has to be modified correspondingly. With the calorimeter, which is to a large extent ready, and a partially equipped vertex detector, the RICH counter is an essential tracking device (see Fig. 5).

The goal of this running period is to measure direct $J / \psi$ production by $920 \mathrm{GeV} / c$ protons, and $B$ production cross section via observing off-thewire $J / \psi$ vertices of $B \rightarrow J / \psi X$. The value of the $B$ production cross-section is of crucial importance for the success of $\mathrm{CP}$ violation measurements. While two experiments have published their results with rather large errors, and theoretical predictions are still accurate only within a factor of two, an independent measurement is urgently needed.

In order to measure the direct $J / \psi$ production, as well as the rate of $J / \psi$ from B decays, the $J / \psi \rightarrow \mathrm{e}^{+} \mathrm{e}^{-}$final state is searched for. In this case, $\mathrm{RICH}$ serves as a device to veto high energetic photons, and thus, enables a considerable reduction of background.

\subsection{Identification of electrons}

In order to identify an electron (or positron), we check whether there are enough Cherenkov

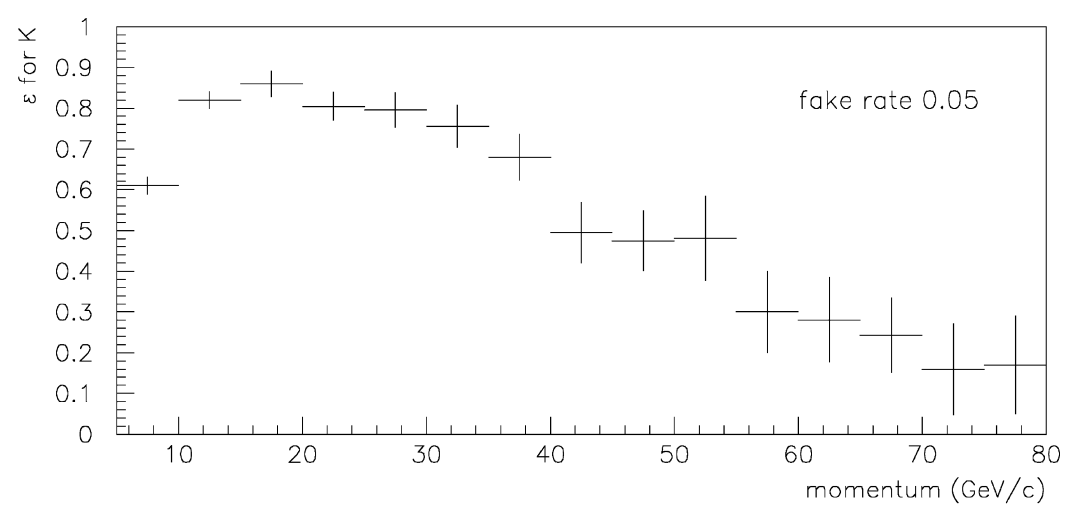

Fig. 2. Kaon identification: expected efficiency at $5 \%$ pion false identification probability. 


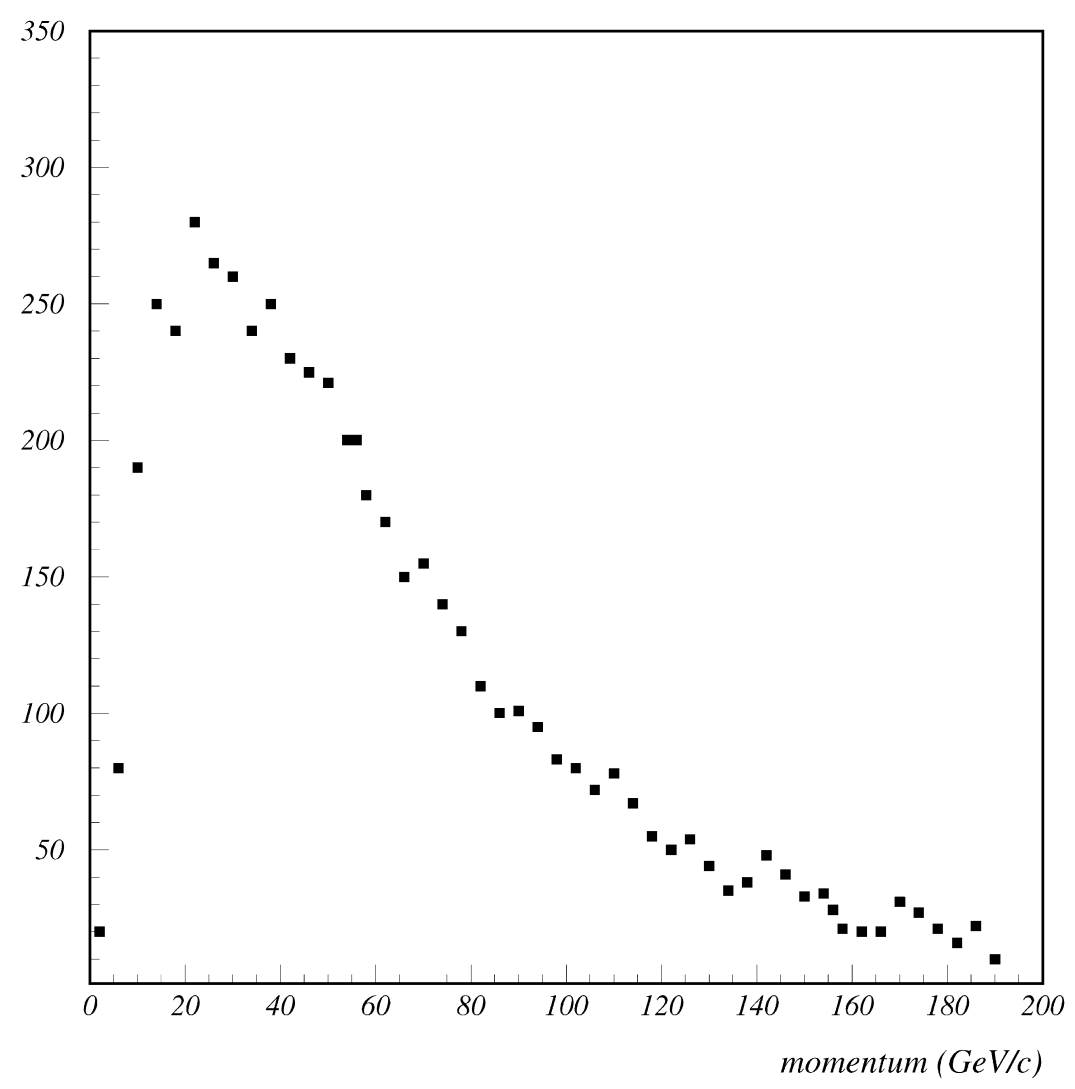

Fig. 3. Momentum spectrum of pions from $B^{0} \rightarrow \pi^{+} \pi^{-}$.

photons associated with the track candidate. When the experiment is operated without a magnetic field, the track direction is deduced with very good accuracy (e.g., $0.3 \mathrm{mrad}$ at $30 \mathrm{GeV} / \mathrm{c}$ ) from the ECAL impact point and interaction point on the target wire. Note that the search for Cherenkov photons is simplified since all electrons have the same (saturated) Cherenkov angle (52 mrad).

A likelihood is calculated for the electron hypothesis, i.e., the $\log$-likelihood difference $\Delta \log L$ of electron + background hypothesis vs. background alone hypothesis. In Fig. 6, this difference is plotted for photons, electrons (both originating in the target and in the material downstream), and other charged particles, mainly pions. A cut at $\Delta \log L>0$ is imposed to select electrons originating in the vicinity of the wire target. For such electrons, the resulting efficiency amounts to about $80 \%$.
The invariant mass distributions for events containing electrons from the $J / \psi$ decay are shown in Fig. 7, before and after applying the RICH identification cut. The cut on electron likelihood eliminates minimum bias events very efficiently (see Fig. 8), and considerably improves the signal-to-noise ratio by reducing the combinatorial background in events containing a $J / \psi \rightarrow \mathrm{e}^{+} \mathrm{e}^{-}$ decay.

A slightly different procedure for identification of electrons and positrons has to be used in case the magnetic field (with a field integral of $2.1 \mathrm{Tm}$ ) is switched on. First the infinite momentum track direction is deduced from the ECAL impact point in the same way as in the field-free case (see Fig. 9). In the second step, a correction is applied to account for the finite bending angle by making use of the energy as measured in the ECAL to estimate the momentum. The bending angle is 

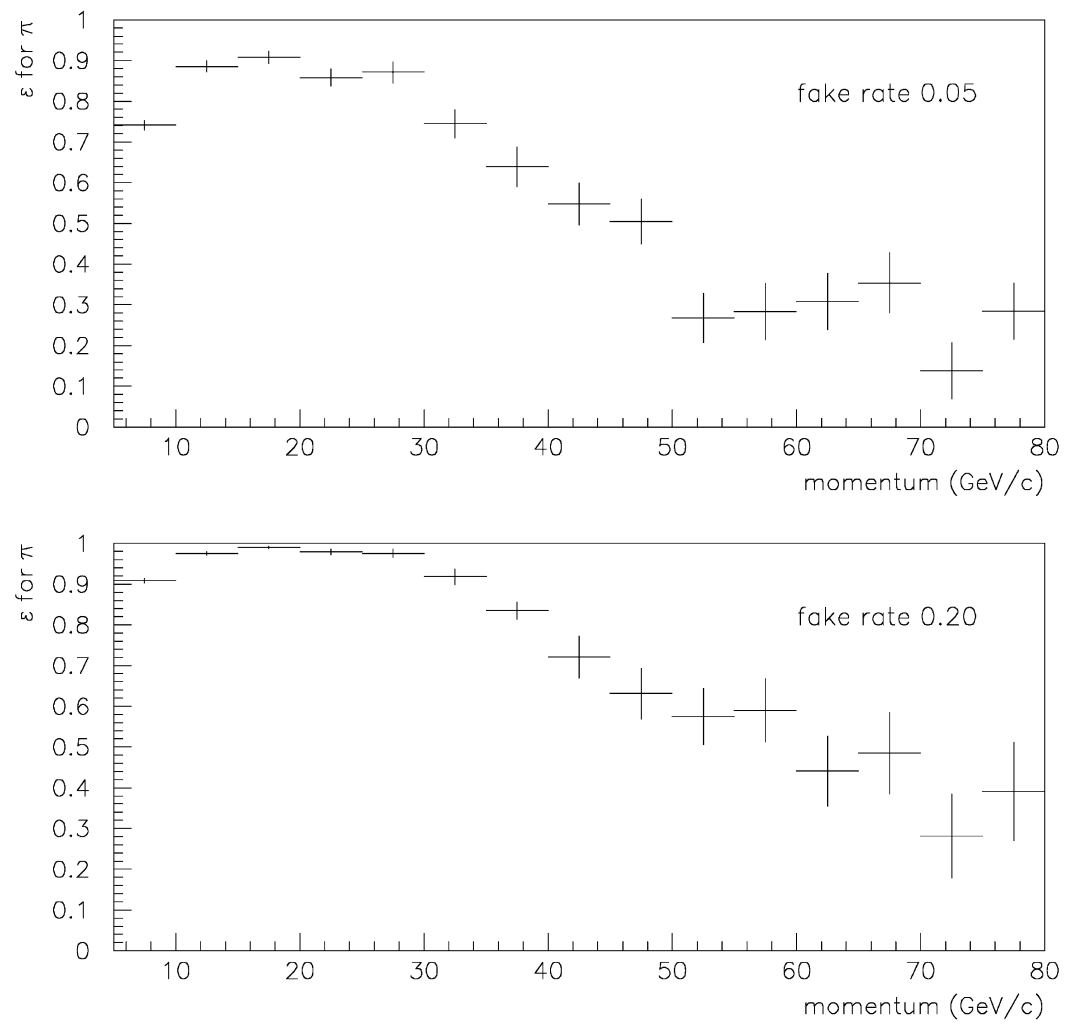

Fig. 4. Pions: expected identification efficiency at $5 \%$ and $20 \%$ kaon false identification probability.

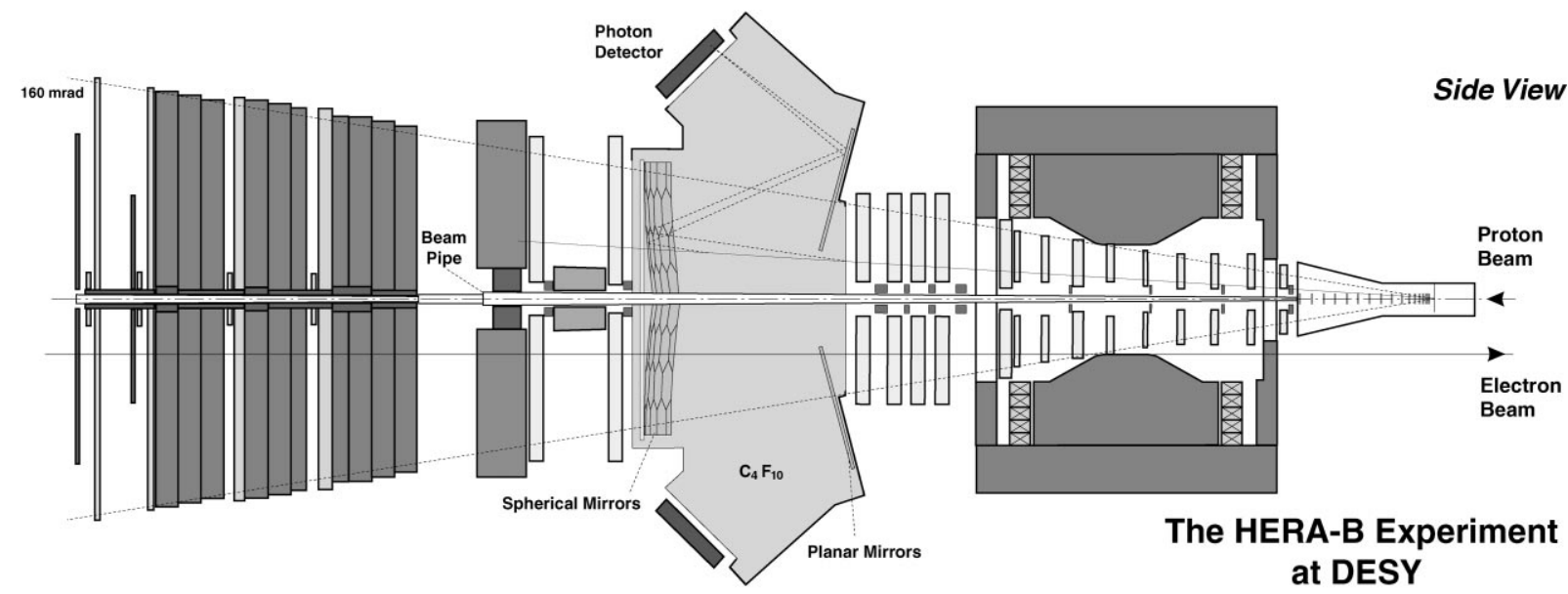

Fig. 5. HERA-B spectrometer, cut in the vertical, non-bending plane. 


\section{4 single $J / \Psi$ events}
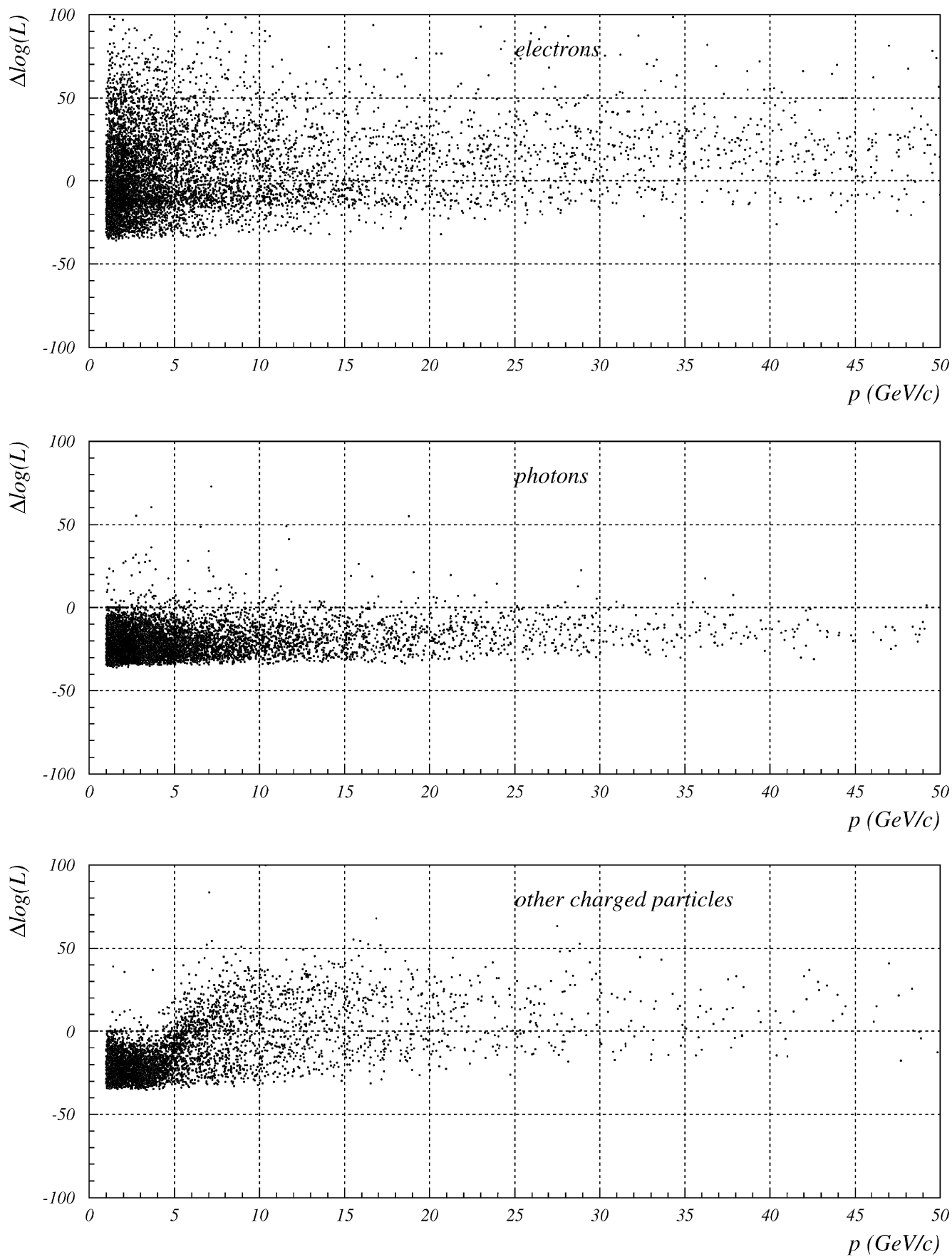

Fig. 6. The log-likelihood difference of electron plus background hypothesis vs. background alone hypothesis, for electrons, photons, and all other charged particles, mainly pions. 


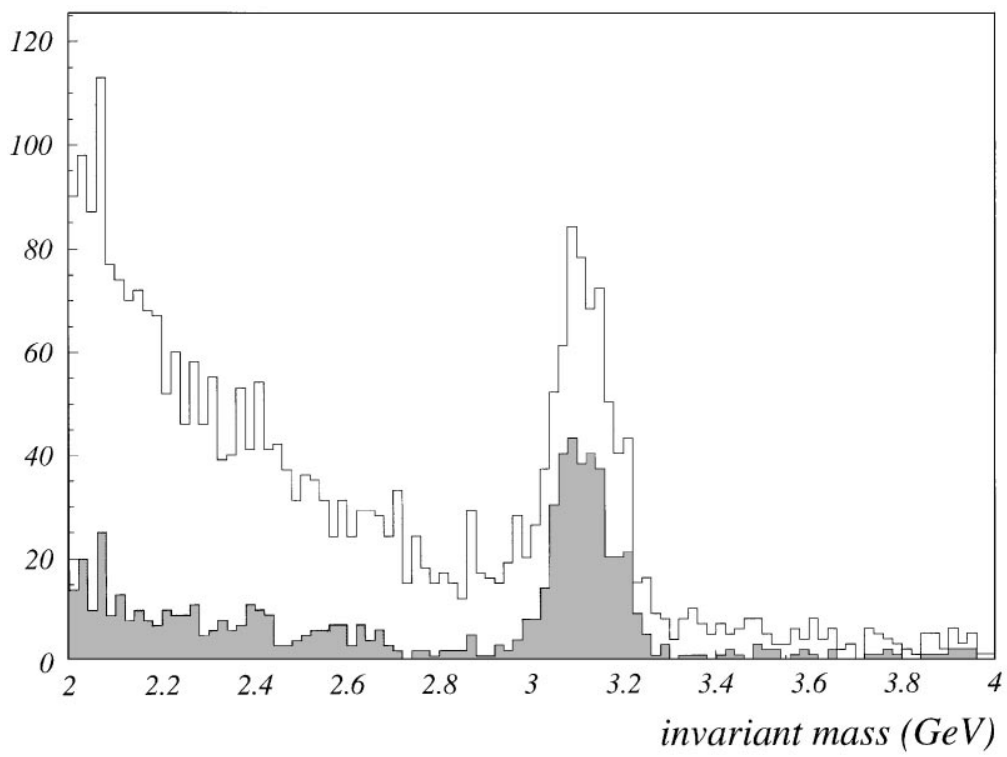

Fig. 7. Invariant mass from pairs of calorimeter clusters in the case of events containing $J / \psi \rightarrow \mathrm{e}^{+} \mathrm{e}^{-}$decays. Open histogram: no cuts, shaded histogram: both clusters are identified in RICH as originating from electrons.

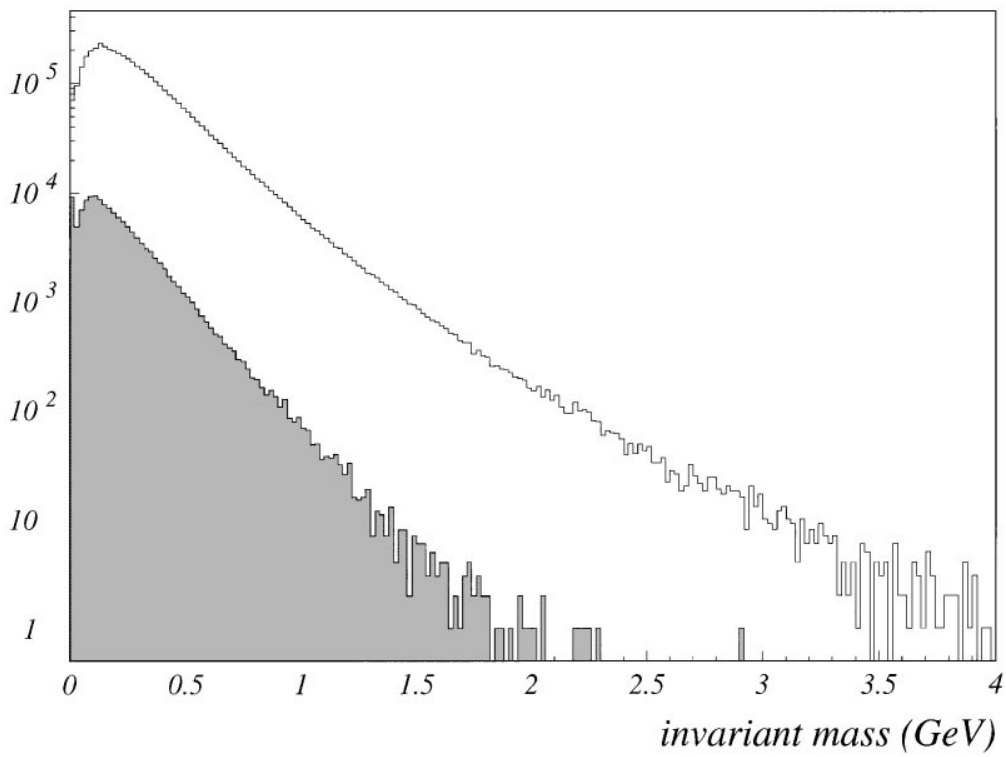

Fig. 8. Same as Fig. 7 for minimum bias inelastic events.

parametrized by

$\phi=\frac{0.635 \mathrm{GeV} / c}{p}$ and the direction at the ECAL (see Fig. 9) differs by $\delta=\frac{0.24 \mathrm{GeV} / c}{p}$ 


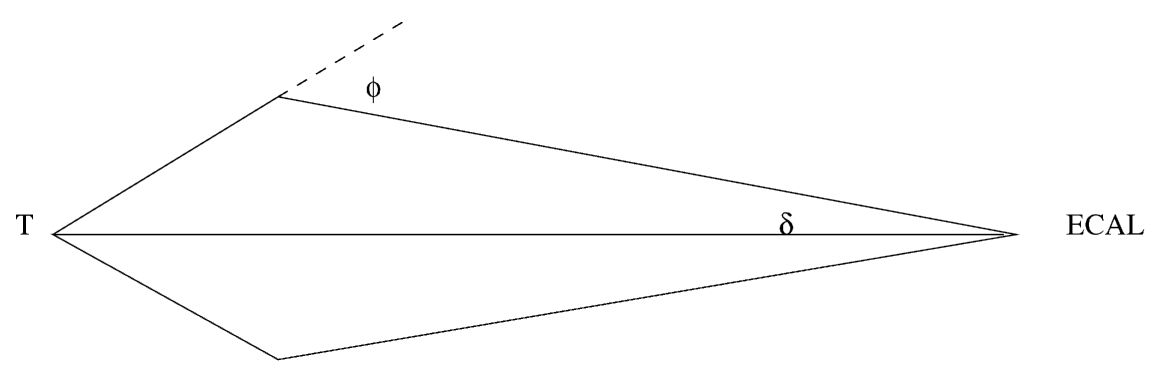

Fig. 9. A simplified picture of the influence of bending on the position of the ring.

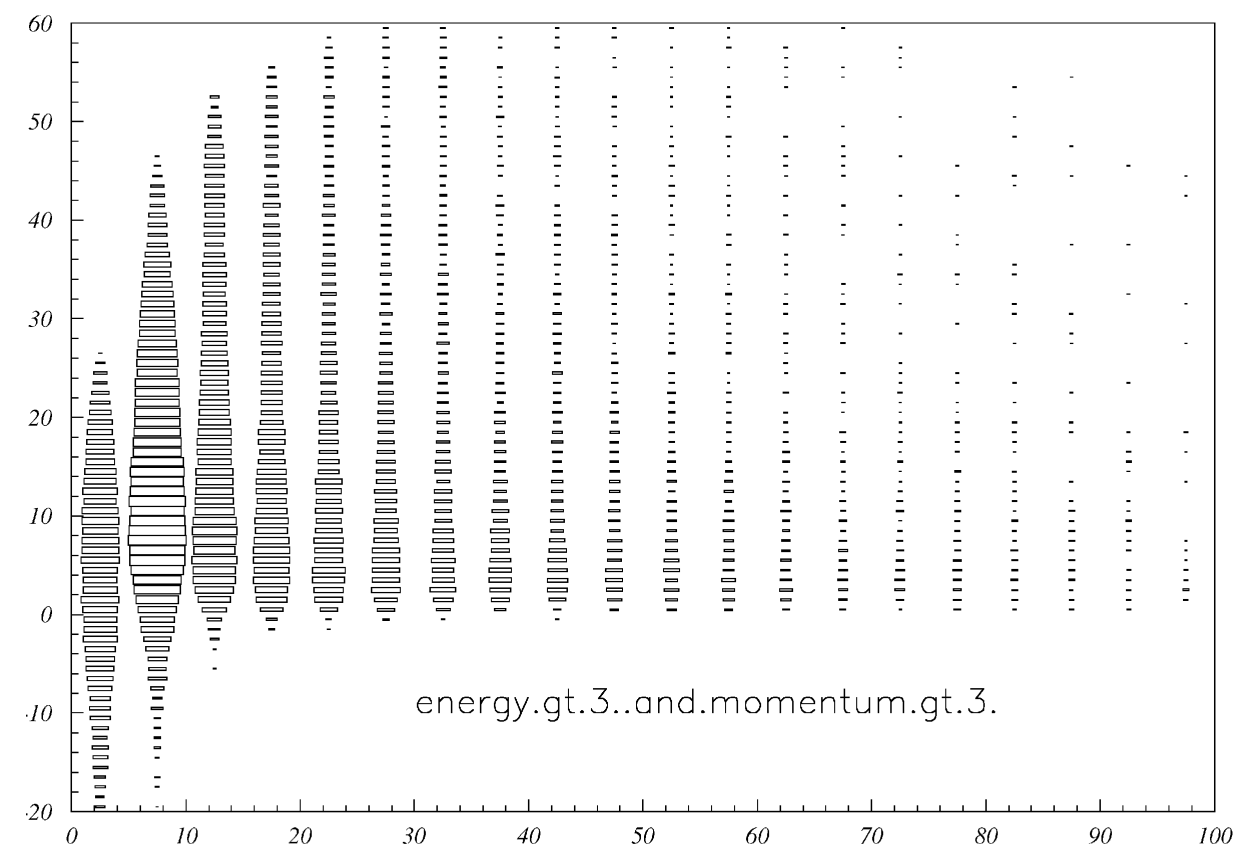

predicted ring position difference for pions ( $\mathrm{mrad}$ ) vs. $p$

Fig. 10. Pion rejection: departure of the ring center from the position as expected from the energy deposited in the calorimeter. Only pions with momentum above $3 \mathrm{GeV} / c$ and energy deposition above $3 \mathrm{GeV}$ were considered in the plot.

from the straight line, connecting the calorimeter impact point with the target. Clearly, since the electron and positron paths are different, we get two solutions. Finally, we look for rings on both the electron side and on the positron side. Since the two rings are well separated (e.g., by about $10 \mathrm{mrad}$ at $40 \mathrm{GeV} / c$ ) when compared with the single photon resolution, a clear distinguishing between the two hypothesis should be possible.

Since pions that have interacted hadronically in the calorimeter deposit there only a fraction of their energy, this deposited energy is a poor measure of pion momentum. Therefore, in the calculation of $\delta$ a wrong value of momentum is used in case of 

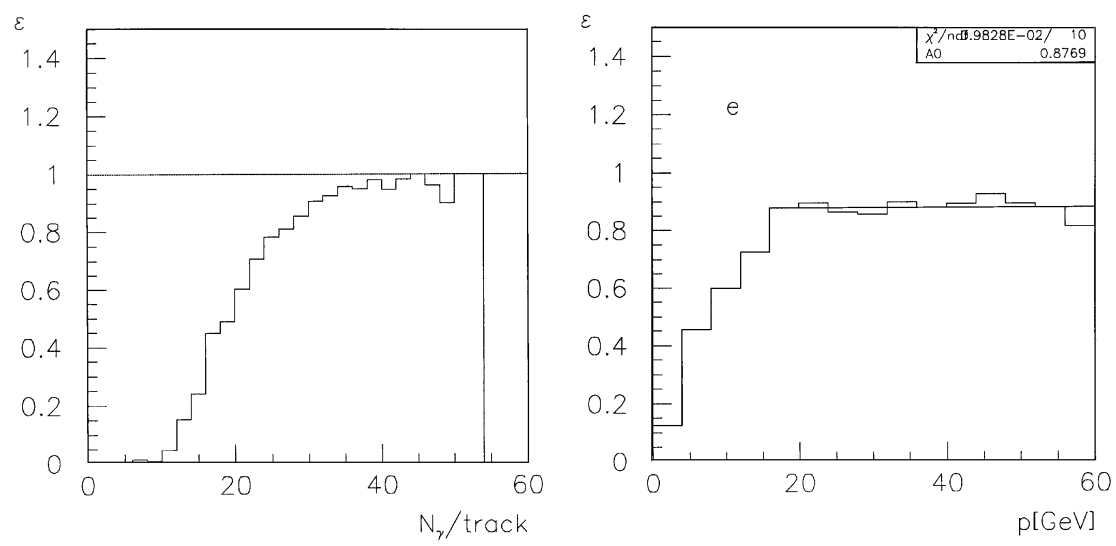

Fig. 11. Ring search efficiency as a function of the number of photons and electron momentum.

a pion, and the ring is searched for at a wrong position. The departure, of course, depends on the momentum, but clearly exceeds the angular resolution of the RICH counter (see Fig. 10). It is thus expected that in this case a good pion/electron separation will be possible over most of the kinematic region covered by the spectrometer.

\section{RICH as a stand-alone tracking device}

$\mathrm{RICH}$ can, in addition, serve as a stand-alone tracking device. From the rings found on the photon detector, track directions are deduced, and matched with the track candidates from the silicon vertex detector.

A stand-alone ring search algorithm is used to identify track candidates with an efficiency as shown in Fig. 11. From the track direction at the position of the RICH (after the magnet) a corresponding track segment is searched for in the vertex detector (before the magnet). First the track segments are matched in the non-bending plane. From the deflection in the bending plane the momentum is then determined.

The expected matching efficiency is about $70 \%$ for low multiplicity events, with an angular resolution below $0.6 \mathrm{mrad}$ for momenta above $20 \mathrm{GeV} / c$. The resulting momentum resolution is slightly below $4 \%$ at $50 \mathrm{GeV} / c$, as can be seen from Fig. 12 . With such a performance of the system, exclusive final states like $\mathrm{B}^{+} \rightarrow J / \psi \mathrm{K}^{+}$become accessible.

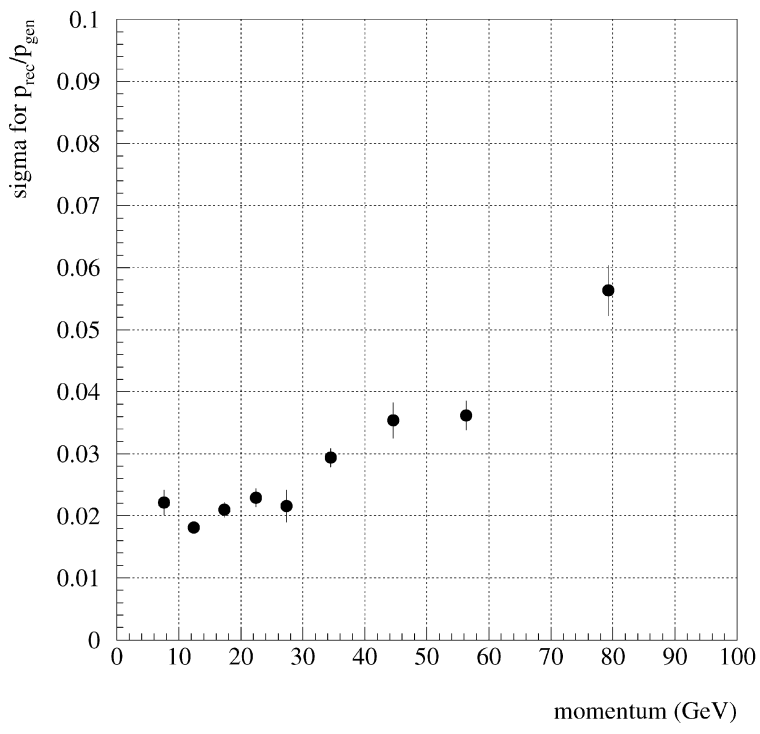

Fig. 12. Momentum resolution for tracks with matched $\mathrm{RICH}$ and vertex detector segments.

\section{Summary}

The estimates of the physics potential of the HERA-B RICH counter are more reliable after the full counter was successfully tested. The expected performance matches well with the momentum distribution of the tagging kaons. The use of the counter as an additional tracking device seems to be promising. This is important for the physics programme in the initial running phase, in 
particular since most of the tracking system will not be available until summer 1999.

\section{References}

[1] T. Lohse et al., Proposal for HERA-B, DESY PRC-94/02, May 1994.
[2] P. Križan et al., Nucl. Instr. and Meth. A 371 (1996) 295.

[3] J.L. Rosen, Nucl. Instr. and Meth. A 408 (1998) 191.

[4] P. Križan et al., IEEE Nuclear Science Symposium and Medical Imaging Conference, November 1997, Albuquerque, NM, Conference Records, pp. 353-356.

[5] S. Korpar et al., Nucl. Instr. and Meth. A 433 (1999) 128. 